\title{
CONSULTING AN EXPERT WITH POTENTIALLY CONFLICTING PREFERENCES
}

May 2007.

\begin{abstract}
We study a situation where a decision maker relies on the report of a selfinterested and informed expert prior to decide whether to undertake a certain project. An important feature in this interaction is that, depending on the collected information, the two agents have potentially conflicting preferences. Information contained in the report is partially verifiable in the sense that the expert can suppress favorable information sustaining the project but he cannot exaggerate it. Our results show that this setting favors the agent which is the less eager to undertake the project in that he always succeeds to induce his most preferred action.
\end{abstract}

KEY WORDS: Information transmission; Verifiable messages; Potentially conflicting preferences

JEL CLASSIFICATION: C72, D82. 


\section{Introduction}

We examine a situation in which a decision maker consults a self-interested and better informed expert prior to decide whether to undertake a certain project. Such a situation is often observed in many economic problems. For instance, for risk management, decision maker relies on scientific expert to evaluate whether an activity is harmful or not as nuclear programs, transgenic crops or new technologies. We consider a communication game where after having received information transmitted by the expert, the decision maker chooses an action that is payoff relevant for both agents (Sender-Receiver game). We consider costless and non-binding information. This paper relies on two fundamentals assumptions. The first one concerns the agents' preferences. We assume that depending on the collected information, the decision maker and the expert have potentially conflicting preferences. That is, for a given information either both agents share the same preferred action (both agents want either the project to be undertaken or not) or they do not (one agent wants it while the other does not). Each agent would like the project to be undertaken if and only if the information is at least as favorable as his own threshold level. Thus, given information may be sufficiently favorable to carry out the project for one agent while it is not for the other. The problem is about the information transmission since the expert is selfinterested and privately informed. Namely, if the expert is less or more eager than the decision maker to undertake the project, the expert might have the incentive to distort his information in order to influence the final decision. Our second fundamental assumption concerns the nature of the reported information. As in Wolinsky $(2002,2003)$ we assume that information collected by the expert is about the desirability of the project and is based on verifiable evidence whereas the absence of it is not verifiable ${ }^{1}$. So, the expert can suppress favorable information about the project whereas he cannot exaggerate it. Thus, in terms of verifiability, the expert can prove that his information is at least as favorable as a given level, but he cannot prove that it is not more.

We develop a simple model with a binary set of actions and we characterize all the equilibria of this interaction. Our results are the followings. Except for the uninformative (or pooling) equilibrium - $\underline{\text { i.e. }}$, in which no relevant information is transmitted - (Proposition 1), we show that the nature of equilibrium is closely linked to the most eager agent. Indeed, when the expert is less eager to undertake the project, due to the potentially conflicting preferences, (generically) all non-pooling equilibria that are monotone - $\underline{i . e .}$, with outcomes satisfying that the more the observed information sustains the project the higher is the probability to undertake it - are such that the expert always succeeds to induce his most preferred action (Proposition 2). Whereas, when the decision maker is less eager to undertake the project, due to the verifiability constraint there always exists equilibrium where the information is fully revealed (Proposition 3). We also show that the existence of non-monotonic equilibrium requires the existence of non-pooling monotonic equilibrium. Furthermore, except for the pooling equilibrium, the decision maker is (generically) better-off ex-ante in any monotonic equilibrium than in any non-monotonic one (Proposition 4).

A well-known result in the literature is that a complete provability setting gives the decision 
maker the opportunity to adopt a skeptical behavior that creates incentives for the expert to fully reveal his private information. Here, due to our setting of partial provability this result only holds when the expert is the more eager to undertake the project. In that case, since the expert cannot exaggerate the favorable information sustaining the project, the decision maker can extract all information at equilibrium by not undertaking the project at least the expert reports him some sufficiently favorable information. On the contrary, when the expert is the less eager agent the outcome of this interaction is comparable to the one where the decision maker would completely delegate the decision-making to the expert. In that case, since the expert cannot prove that he is not withholding some favorable information the communication becomes similar to one without any provability (cheap-talk). And then as depending on the information both agents may often share the same preferred action (in particular without uncertainty both agents would agree), the decision maker totally trusts the expert.

Related Works. There exists a substantial literature considering strategic information transmission with verifiable information between a self-interested expert and an uninformed decision maker.

This literature can be separated into four broad categories according to whether or not a decision maker faces an informed party that has monotonic preferences - $\underline{\text { i.e. }}$, that wants the decision maker to maximize (or minimize) the magnitude of his action - and/or the ability to prove all decision-relevant information.

Most of this work focuses on situations where a decision maker faces an informed party that has both monotonic preferences and ability to prove all his decision-relevant information (e.g., Grossman (1981), Grossman and Hart (1980), Koessler (2003), Matthews and Postlewaite (1985), Milgrom (1981), Milgrom and Roberts (1986)). The central result called as the unraveling argument (demonstrated in a general setting by Seidmann and Winter (1997)) is that at equilibrium, by using a skepticism strategy the decision maker succeeds to fully extract the informed party's private information.

Some models consider the case of an informed party with monotonic preferences in a setting of partial provability. Okuno-Fujiwara et al. (1990) show that the unraveling argument extends to a situation where the decision maker knows that the informed party wants to maximize the magnitude of his decision and is able to prove that the observed information is at least as favorable than a certain threshold ${ }^{2}$. Shin $(1994 a, 1994 b)$ provides a model in which the expert is unable to prove the precision of his information. A skeptical inference then might be irrational and the author shows that there is no fully revealing equilibrium. Wolinsky (2003) considers a situation where the decision maker does not know whether the expert is of a type that wants to maximize or minimize the magnitude of a certain action. In this setting, he characterizes a unique equilibrium outcome as a combination of the equilibria that would prevails in the certainty expert's preferences world.

Some models of complete provability allow the expert's preferences to depend on his private information (non-monotonic preferences). When the expert can always prove his information, Seidmann and Winter (1997) derive necessary conditions for the existence of a fully revealing equilibrium and sufficient conditions for its existence and uniqueness. When in addition the ex- 
pert can prove any true event, Giovannoni and Seidmann (2006) provide necessary and sufficient conditions for the existence of a separating equilibrium and/or a pooling equilibrium.

Finally, only recent literature deals with situation where the expert's most preferred action depends on his decision-relevant private information in a setting of partial provability. Mathis (2007) generalizes Seidmann and Winter's (1997) results to the partial provability setting (e.g., expert that is unable to prove all the information he has observed or that he is not withholding other information). Wolinsky (2002) considers a model with multiple experts who share similar preferences that always are in conflict with the decision maker's preferences. The author compares cases where the decision maker may allow communication among them and whether have or not commitment ability. In particular, he shows that in the case of no commitment and no communication, due to the fact that experts understand that their reports matter only when they are pivotal, no information is revealed in equilibrium.

The remainder of this paper is organized as follows. Section 2 describes the model. Section 3 characterizes the equilibrium outcome of this interaction.

\section{The Model}

The communication game. A decision maker, DM, has to choose an action $a \in\{x, y\}$. The choice of the action $a$ depends on a information $v$ that DM does not have. The way we formalize verifiable information is similar to Wolinsky (2003). More precisely, we assume the following. The information $v \in\left\{v_{1}, v_{2}, \ldots, v_{N}\right\}$, where $v_{1}<v_{2}<\ldots<v_{N}$. The prior distribution of $v$ has full support, $\operatorname{Pr}\left(v=v_{n}\right)>0$ for all $n$. DM consults an expert, E, who observes $v$ and reports $r \in\left\{v_{1}, v_{2}, \ldots, v_{N}\right\}$ before the decision is made. The report is partly verifiable in the sense that E can report $r=v_{i}$ only when the true value is $v_{n} \geq v_{i}$. The timing of the communication game is represented by Figure 1 .

\begin{tabular}{|c|c|c|}
\hline Information phase & Talking phase & Action phase \\
\hline $\begin{array}{c}\text { Expert learns } \\
v_{i} \in\left\{v_{1}, v_{2}, \ldots, v_{N}\right\}\end{array}$ & $\begin{array}{r}\text { Expert reports } \\
r \in\left\{v_{1}, v_{2}, \ldots, v_{i}\right\}\end{array}$ & $\begin{array}{c}\text { DM chooses action } \\
a \in\{x, y\}\end{array}$ \\
\hline
\end{tabular}

Figure 1: The timing of the communication game

Preferences. The agents' preferences depend on the action $a$ and the collected information $v$. The higher is $v$ the more $v$ is conveying favorable information about the action $y$. More precisely, the agent $j$ 's preferences, with $j \in\{D M, E\}$, is a function $u^{j}:\{x, y\} \times\left\{v_{1}, v_{2}, \ldots, v_{N}\right\} \mapsto \mathbb{R}$ where $u^{j}(a, v)$ denotes the $j$ 's utility level when action $a$ is taken and $v$ is realized. We suppose $u^{j}(x,$.$) decreasing in v$ and $u^{j}(y,$.$) increasing in v$. Moreover, we assume $u^{j}\left(x, v_{1}\right)>u^{j}\left(y, v_{1}\right)$ and $u^{j}\left(x, v_{N}\right)<u^{j}\left(y, v_{N}\right)$. From these assumptions, we can characterize a threshold $q^{j} \in\left(v_{1}, v_{N}\right)$, where for all $n, u^{j}\left(y, v_{n}\right)>u^{j}\left(x, v_{n}\right) \Leftrightarrow v_{n}>q^{j}$ and $u^{j}\left(y, v_{n}\right)<u^{j}\left(x, v_{n}\right) \Leftrightarrow v_{n}<q^{j}$. With this formulation, conflicting preferences between the two agents occurs when E observes a $v$ which is between the two thresholds $q^{D M}$ and $q^{E}$. For convenience, we assume that for any value, both 
agents have a strictly preferred action, that is $q^{j} \neq v_{n}$ for any $n, j=E, D M$. Figure 2 gives the full information benchmark for players' preferences.

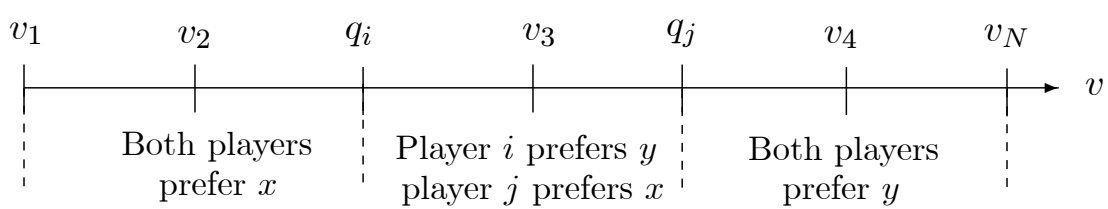

Figure 2: Full information benchmark

Players' strategies. A (mixed) strategy for E is a conditional distribution describing the probability with which $\mathrm{E}$ will report $v_{i}$ when he has observed the value $v_{n}$. E's strategy is denoted $\mu=\left(\mu_{n}^{i}\right)$, where $\mu_{n}^{i}=\operatorname{Pr}\left(r=v_{i} \mid v=v_{n}\right), n=1, \ldots, N, i \leq n$. A (mixed) strategy for DM is a conditional distribution describing the probability with which DM will choose the action $y$ when he receives the report $r$. DM's strategy is denoted $\alpha=\left(\alpha^{i}\right)$, where $\alpha^{i}=\operatorname{Pr}\left(a=y \mid r=v_{i}\right)$, $i=1, \ldots, N$. Let $p_{n}^{i}(\mu)$ describes DM's belief that $v=v_{n}$ conditional on a report $r=v_{i}$ when E reports according to the strategy $\mu$. If for some $m, \mu_{m}^{i}>0$ then the conditional probability $\operatorname{Pr}\left(v=v_{n} \mid r=v_{i}, \mu\right)$ is well defined and $p_{n}^{i}(\mu)=\operatorname{Pr}\left(v=v_{n} \mid r=v_{i}, \mu\right)$; if $\mu_{m}^{i}=0$ for all $m$, then the only restriction on $p_{n}^{i}(\mu)$ is that it is consistent with the verifiability assumption, i.e., $p_{n}^{i}(\mu)>0$ only if $i \leq n$. Following a report $r=v_{i}$, the expected value of $v$ given DM's beliefs is

$$
E\left[v \mid r=v_{i}, \mu\right]=\sum_{m=i}^{N} v_{m} p_{m}^{i}(\mu)
$$

and the expected DM's utility of taking action $a$ given DM's beliefs is

$$
E\left[u^{D M}(a, .) \mid r=v_{i}, \mu\right]=\sum_{m=i}^{N} u^{D M}\left(a, v_{m}\right) p_{m}^{i}(\mu)
$$

We will equivalently say that DM's action $a$ will be the best response to the report $r=v_{i}$ under E's strategy $\mu$ or his preferred action according to the expected value $E\left[v \mid r=v_{i}, \mu\right]$ if $E\left[u^{D M}(a,) \mid. r=v_{i}, \mu\right] \geq E\left[u^{D M}\left(a^{\prime},.\right) \mid r=v_{i}, \mu\right]$ for any action $a^{\prime}$.

Equilibrium. An equilibrium is a pair $(\mu, \alpha)$ such that

$$
\mu_{n}^{i}>0 \text { implies }\left\{\begin{array}{c}
i \in \underset{k \leq n}{\arg \max } \alpha^{k} \text { if } v_{n}>q^{E} \\
i \in \underset{k \leq n}{\arg \min } \alpha^{k} \text { if } v_{n}<q^{E}
\end{array},\right.
$$

$\alpha^{i}=1$ implies the action $y$ is a DM's preferred action according to $E\left[v \mid r=v_{i}, \mu\right]$, and $\alpha^{i} \in(0,1)$ implies both actions are preferred (according to $E\left[v \mid r=v_{i}, \mu\right] \mathrm{DM}$ is indifferent between $x$ and $y$ ).

Thus, E's equilibrium strategy is such that when a $v_{n}>q^{E}\left(\underline{\text { resp. }} . v_{n}<q^{E}\right)$ is observed, E reports with positive probability only $v_{i}$ 's that maximizes (resp. minimizes) the probability 
that DM chooses the action $y$, subject to the constraint $r \leq v_{n}$. DM's equilibrium strategy is such that, following a report $r=v_{i} \leq v_{n}$, DM's action $a$ is a preferred action according to the expected value $E\left[v \mid r=v_{i}, \mu\right]$ given DM's belief. Note that the equilibrium definition embodies the requirement that DM's belief is rational (i.e. $p_{n}^{i}():.=\operatorname{Pr}\left(v=v_{n} \mid r=v_{i},.\right)>0$ only if $\left.i \leq n\right)$.

\section{Results}

This section characterizes the equilibrium outcome of this interaction. The first three propositions deal with the monotonic equilibria - that is, the equilibria which have the property that higher values are always associated with higher probability to choose the action $y$. From now on, we shall say that at equilibrium a value $v$ induces the action $y$ (resp. $x$ ) (without specifying any probability) if after having observed any report sent with positive probability by an expert with value $v, \mathrm{DM}$ chooses action $y$ (resp. $x$ ) with probability 1 in that equilibrium. Due to the verifiability assumption, a report $r$ proves that the true value $v$ is greater or equal than $r$. For any report $r>q^{D M}$, DM's best response is then $y$. A simple and useful property on the equilibria outcomes is the following Lemma.

Lemma 1. Any equilibrium outcome satisfies the following:

i) Any value $v>\max \left\{q^{E}, q^{D M}\right\}$ induces the action $y$.

ii) If there is a report $r<\min \left\{q^{E}, q^{D M}\right\}$ for which DM's best responds by $x$ then any value $v \in\left[r, q^{E}\right)$ will induce the action $x$.

Lemma $1 \mathrm{ii}$ ) gives a sufficient condition for the existence of a value $v$ which at equilibrium, induces the action $x$. Proposition 1 gives the necessary and sufficient condition for the absence of a value $v$ which induces the action $x$ with positive probability. Observes that this condition does not depend on which is the more eager agent to undertake the project.

Proposition 1. There exists a uninformative equilibrium (which always induces the action $y)$ iff the DM's preferred action according to $E(v)$ is $y$.

Straightforward to Lemma $1 i$ ), a pooling equilibrium where action $x$ is always taken is not possible since an expert with information $v>\max \left\{q^{E}, q^{D M}\right\}$ wants and can convince DM to take action $y$, by simply reporting $r=v$. However, an expert who wants DM to take action $x$ is not always able to report a convincing message since the absence of information is not verifiable. So, a pooling equilibrium may exist where action $y$ is always taken as in Proposition 1. Of course, existence of such an equilibrium relies on that DM's preferred action according to $E(v)$ is $y$.

Proposition 2 deals with the monotonic equilibria where $\mathrm{E}$ is less eager than DM to undertake the project. The footnote of this proposition exhibits a mixed equilibrium outcome which generically does not exist as it will be shown in the proof of Proposition 4 .

Proposition 2. Suppose $q^{D M} \leq q^{E}$. There exists an equilibrium in which the expert always succeeds to induce his most preferred action iff the DM's preferred action according to $E\left(v \mid v<q^{E}\right)$ is $x .^{3}$ 
Proposition 2 states that in addition to the pooling equilibrium, when $\mathrm{E}$ is less eager than $\mathrm{DM}$, there may exist another monotonic equilibrium in which $\mathrm{E}$ always succeeds to induce his most preferred action. Here the existence of such equilibrium does not rely on the verifiability assumption. In particular, the direction of the verifiability does not allow DM to compel E to reveal his information. Instead, this existence owes to both the fact that E's most preferred action depends on his observed value $v$ and that the agents may share the same preferred action. Namely when E observes a value $v$ he sends a report which maximizes the probability to induce the action $y$ if $v>q^{E}$ and a report which minimizes it if $v<q^{E}$. When DM receives such a report he then learns either if the value is greater or smaller than $q^{E}$. In the first case, both agents share $y$ as their preferred action while in the second case, the condition for DM to choose $x$ is that it is his preferred action according to $E\left(v \mid v<q^{E}\right)$ as exhibited in Proposition 2.

When there exists a report $r$ for which DM best responds by $x$, the optimality of E's equilibrium strategies implies that $\mathrm{E}$ will induce the action $x$ when $r \leq v<q^{E}$. Observe that such an equilibrium is monotonic only if it also induces the action $x$ when $v<r$, in particular when $v=v_{1}$. So, the report $r=v_{1}$ must induces the action $x$. Thus, the expert always succeeds to induce his most preferred action by reporting $r=v_{1}$ when $v<q^{E}$ and $r \geq v$ else. Otherwise, when there does not exist such a report for which DM best responds by $x$, the monotonic equilibrium is either the pooling one characterized in Proposition 1 or these of the footnote. This latter exists only if $q^{D M}=E\left(v \mid v<q^{E}\right)$, a condition which generically does not hold.

For notation convenience, we have assumed that $q^{j} \in\left(v_{1}, v_{N}\right), j=D M, E$. This without loss of generality since our equilibria still hold if $q^{j} \in\left[v_{1}, v_{N}\right]$. Suppose that $q^{E}=v_{N}$, whatever the collected information, the most preferred action of $\mathrm{E}$ will always be the action $x$. Thus, as suggested by the related literature, the unique equilibrium is pooling which induces either the action $y$ (Proposition 1) or the action $x$ (Proposition 2) depending on which action is the DM's preferred action according to $E(v)$. Observe that the footnote of Proposition 2 deals with a mixed equilibrium which generically does not exist.

Proposition 3 deals with the monotonic equilibria where $\mathrm{E}$ is more eager than DM.

Proposition 3. Suppose $q^{E} \leq q^{D M}$. There always exists a fully revealing equilibrium. More generally, there exists a continuum of equilibria, parameterized by $q \in\left[q^{E}, q^{D M}\right]$, which induce the action $x$ when $v \leq q$ and the action $y$ else iff the DM's preferred action according to $E(v \mid v>q)$ is $y$.

In such a situation, as suggested by the existing literature (Grossman (1981), Grossman and Hart (1980), Milgrom (1981)), DM can totally extract information from E by using a skepticism strategy - that is here, a strategy in which DM takes the action $x$ unless E convince him by reporting a sufficiently high report $r \geq q^{D M}$. In this manner the full information decision Milgrom Roberts (1986) - the decision that would have been reached if the decision maker had perfect access to the private information of the expert - occurs. Since by definition, DM's preferred action according to $E\left(v \mid v \geq q^{D M}\right)$ is $y$, this fully revealing equilibrium outcome always exists. In addition here some equilibrium also may exist in which DM can partially extract information from E by using what we call a weakened skepticism strategy. That is a strategy in 
which DM is less compelling in the sense that rather than taking the action $x$ unless $\mathrm{E}$ reports a report $r \geq q^{D M}$, DM takes the action $x$ unless $\mathrm{E}$ reports a report $r \geq q$, with $q<q^{D M}$. The level of skepticism is parameterized by $q$. The lower is $q$, the less DM is skeptic. The condition on the existence of such equilibria is that DM's preferred action according to $E(v \mid v \geq q)$ is $y$. At equilibrium, this is the verifiability assumption which allows DM to uses some strategies relying on skepticism. The possibly uses of weakened skepticism at equilibrium is also due to the fact that for all value $v>q^{E}$ E has the same preferred action $y$. So by considering two different values $\tilde{v}_{1}, \tilde{v}_{2}$, with $\tilde{v}_{1}>\tilde{v}_{2}>q^{E}$, this is not costly for $\tilde{v}_{1}$ to send the same report $r$ than $\tilde{v}_{2}$, as long as $r$ induces the action $y$.

Proposition 4 deals with the others equilibria outcomes of the game.

Proposition 4. All other equilibria outcomes are non-monotonic. The conditions for their existence are more restrictive than those for the non-pooling monotonic ones. Furthermore, except for the pooling equilibrium, DM is (generically) better-off ex-ante in any monotonic equilibrium than in any non-monotonic one.

According to Proposition 4, this game does not admit others monotonic equilibria outcomes than those exhibited in the first three propositions. That is, if there is another equilibrium outcome then it is such that there are two values $v_{k}$ and $v_{l}$, with $v_{k}<v_{l}$, satisfying that the probability to choose action $y$ is strictly higher under $v_{k}$ than under $v_{l}$. Such equilibrium outcome may exist even when players have aligned preferences as illustrated by the following simple numerical example. Let $N=3$ with $\operatorname{Pr}\left(v_{1}\right)=\operatorname{Pr}\left(v_{2}\right)=\frac{1}{4}$ and $\operatorname{Pr}\left(v_{3}\right)=\frac{1}{2}$; and both players having same preferences represented by $u\left(x, v_{1}\right)=u\left(y, v_{3}\right)=1, u\left(y, v_{1}\right)=u\left(x, v_{3}\right)=0$, $u\left(x, v_{2}\right)=\frac{2}{3}$ and $u\left(y, v_{2}\right)=\frac{1}{3}$. Now consider that DM chooses $x$ if $r=v_{2}$ and $y$ else; and that $\mathrm{E}$ reports $r=v_{1}$ if $v \in\left\{v_{1}, v_{3}\right\}$ and $r=v_{2}$ if $v=v_{2}$. Under such reporting strategy, DM's updated beliefs are given by $p_{1}^{1}=\frac{1}{3}, p_{3}^{1}=\frac{2}{3}$ and $p_{2}^{2}=1$. These strategies constitute an equilibrium that induces the action $y$ when $v \in\left\{v_{1}, v_{3}\right\}$, and $x$ when $v=v_{2}$. As it will be shown (see Claim and proof of Proposition 4 in Appendix), the existence of non-monotonic equilibrium outcome requires that there is a parameter $q^{\prime} \leq \min \left\{q^{E}, q^{D M}\right\}$ such that DM's preferred action according to $E\left(v \mid v \in\left[q^{\prime}, q^{E}\right]\right)$ is $x$ and according to $E\left(v \mid v \notin\left[q^{\prime}, q^{E}\right]\right)$ is $y$. These conditions are more restrictive than those of the equilibria depicted in Proposition 2 and 3. To see this, observes that if DM's preferred action according to $E\left(v \mid v \in\left[q^{\prime}, q^{E}\right]\right)$ is $x$ then $x$ is also his preferred action according to $E\left(v \mid v \leq q^{E}\right.$ ) (as in Proposition 2). Similarly, if DM's preferred action according to $E\left(v \mid v \notin\left[q^{\prime}, q^{E}\right]\right)$ is $y$ then action $y$ is still preferred according to $E\left(v \mid v>q^{E}\right)$. Observe that this latter condition is the more restrictive condition on the parameter $q$ exhibited in Proposition 3.

\section{Conclusion}

The contribution of this paper is the characterization of equilibrium in a information transmission game with partially verifiable reports and players' preferences which depends on the information collected by the expert. In this setting, we provide a intuitive result: Depending 
on the acceptability to undertake the project and the verifiability constraint, either the decision maker or the expert succeed to induce his preferred action. When the decision maker is more skeptic to undertake the project, due to verifiability constraint a fully revealing equilibrium always exists. Conversely, when the expert is more skeptic to undertake the project, verifiability constraint has no force and the equilibrium is similar to the issue of a perfect delegation setting.

\section{ACKNOWLEDGMENTS}

Financial support from the French Ministry of Research (Actions Concertées Incitatives) is gratefully acknowledged. We have benefited from insight comments from Françoise Forges, Michel Le Breton, Michèle Cohen, Frédéric Koessler, Regis Renault, Jean-Marc Tallon, Alain Trannoy, and an anonymous referee. We also thank the Game Theory Seminar at Institut Henri Poincaré (Paris), Maison des Sciences Economiques (Paris), CREST-LEI (Paris), and the University of Cergy-Pontoise for helpful comments and discussions.

\section{APPENDIX}

Proof of Lemma 1. i) If $v>\max \left\{q^{E}, q^{D M}\right\}$ then E prefers the action $y$ while he can convince DM to take it by reporting $r=v$.

ii) For all value $v<q^{E}$, E prefers the action $x$. If there is a report $r<\min \left\{q^{E}, q^{D M}\right\}$ for which DM's best responds by $x$ then for all value $v \geq r$, E can induce the action $x$ by simply reporting $r$.

Proof of Proposition 1. Sufficiency. Consider the players' strategy in which E reports $r=v_{1}$ regardless of $v$ and DM ignores E's report and chooses the action $y$.

Necessity. Suppose not: there is a uninformative equilibrium whereas DM's preferred action according to $E(v)$ is not $y$. By definition, a uninformative equilibrium induces the same action for any value $v$. For Lemma $1 i$ ) this action is $y$. Observe that as a rational belief, $E(v)$ (which is the expected value of $v$ with respect to the prior) satisfies the martingale property. Hence, there must exists a value under which with positive probability E makes a report to which DM's best responds by $x$. This leads to a contradiction.

Proof of Proposition 2. Sufficiency. Suppose that $x$ is a DM's preferred action according to $E\left(v \mid v<q^{E}\right)$. The following players' strategies support the equilibrium described in the proposition. According to $v$, E reports $r=v_{1}$ if $v \leq q^{E}$ and reports $r=v$ else. DM chooses the action $x$ if $r=v_{1}$ and chooses the action $y$ else.

Necessity. Suppose not: there is an equilibrium in which the expert always succeeds to induce his most preferred action whereas the DM's preferred action according to $E\left(v \mid v<q^{E}\right)$ is not $x$. Under such an equilibrium, there is no common report $r$ which is sent with positive probability by both an expert with a value $v<q^{E}$ and a value $v>q^{E}$. So, on receipt of a 
report, DM learns whether $v<q^{E}$ or $v>q^{E}$. Since $q^{D M} \leq q^{E}$, DM best responds to $v>q^{E}$ by $y$. Now, since DM's preferred action according to $E\left(v \mid v<q^{E}\right)$ is not $x$, the martingale property implies that there must is a value $v<q^{E}$ under which with positive probability E makes a report to which DM best responds not by $x$, a contradiction.

In the special case of the footnote, for sufficiency just changes the DM's strategy with DM chooses the action $x$ with a fixed probability when $r=v_{1}$.

Proof of Proposition 3. Sufficiency. Fix a $q \in\left[q^{E}, q^{D M}\right]$. Suppose that DM's preferred action according to $E(v \mid v>q)$ is $y$. The following players' strategies support the equilibria described in the proposition. According to $v$, E reports $r=v_{1}$ if $v \leq q$ and reports $r=\min \left\{v_{i} \in\right.$ $\left.\left\{v_{1}, v_{2}, \ldots, v_{N}\right\} \mid v_{i}>q\right\}$ else. DM chooses the action $y$ if $r>q$ and chooses the action $x$ else.

Necessity. Suppose not: there exists an equilibrium, parameterized by $q \in\left[q^{E}, q^{D M}\right]$, which induces the action $x$ when $v \leq q$ and the action $y$ else whereas the DM's preferred action according to $E(v \mid v>q)$ is not $y$. Under such an equilibrium, there is no common report $r$ which is sent with positive probability by both an expert with a value $v \leq q$ and a value $v>q$. So, after he has received a report, DM learns whether $v \leq q$ or $v>q$. Since $q \leq q^{D M}$ DM best responds to $v \leq q$ by $x$. Now, since DM's preferred action according to $E(v \mid v>q)$ is not $y$, the martingale property implies that there must exists a value $v>q$ under which with positive probability $\mathrm{E}$ makes a report to which DM best responds not by $y$. This leads to a contradiction.

The following Lemma establishes some properties on the mixed equilibria we shall need before to prove Proposition 4.

Lemma 2. i) If $q^{E} \leq q^{D M}$ then there is no mixed equilibrium outcomes (i.e., any value induces the action $y$ with either probability 0 or 1 ).

ii) If there is a mixed equilibrium outcomes then there exists a unique mixed distribution over the actions set $\{x, y\}$ (i.e., if there exist two values $\tilde{v}_{1}$ and $\tilde{v}_{2}$ which induce the action $y$ with respectively probability $\beta_{1} \in(0,1)$ and $\beta_{2} \in(0,1)$ then $\left.\beta_{1}=\beta_{2}\right)$.

Proof of Lemma 2. i) Suppose $q^{E} \leq q^{D M}$. At equilibrium, by Lemma $1 i$ ) any value $v>q^{D M}$ induces the action $y$. Therefore, there is no common report $r$ inducing with positive probability the action $x$ which is sent (with positive probability) by both an expert with value $v>q^{D M}$ and with value $v<q^{D M}$.

ii) Suppose not: there exists a mixed equilibrium with two such values $\tilde{v}_{1}$ and $\tilde{v}_{2}$ with $\beta_{1}$ and $\beta_{2}$ satisfying $0<\beta_{1}<\beta_{2}<1$. At equilibrium, for any value $\tilde{v}_{i}, i=1,2$, inducing a mixed distribution over the actions set, there exist another value $v_{i}^{\prime}$ satisfying $\min \left\{\tilde{v}_{i}, v_{i}^{\prime}\right\} \leq q^{D M} \leq$ $\max \left\{\tilde{v}_{i}, v_{i}^{\prime}\right\}$, for which there is a common report $r_{i}$ sent (with positive probability) by both values $\tilde{v}_{i}$ and $v_{i}^{\prime}$. In addition, by Lemma $\left.2 i\right) q^{E}>q^{D M}$. So, by Lemma $\left.1 i\right) \tilde{v}_{1}$ and $\tilde{v}_{2}$ are both smaller than $q^{E}$. But this together with the optimality of E's strategy implies that any report sent with positive probability by an expert with value $\tilde{v}_{i}$ induces the action $y$ with probability $\beta_{i}$. Now, from $\beta_{i}<1$ again by Lemma $1 i$ ) we obtain that $v_{1}^{\prime}$ and $v_{2}^{\prime}$ are both smaller than $q^{E}$. By assumption there is no $\tilde{v}_{i}=q^{E}$, we then have $\max \left\{\tilde{v}_{1}, \tilde{v}_{2}, v_{1}^{\prime}, v_{2}^{\prime}\right\}<q^{E}$. The fact that 
$\beta_{1}<\beta_{2}$ implies that the report $r_{1}$ is not available to $\mathrm{E}$ with a value $\tilde{v}_{2}$ or $v_{2}^{\prime}$. Therefore from the direction of the verifiability $\max \left\{\tilde{v}_{2}, v_{2}^{\prime}\right\}<\min \left\{\tilde{v}_{1}, v_{1}^{\prime}\right\}$, a contradiction.

Proof of Proposition 4. We proceed in five steps. First, we prove that all monotonic equilibria outcomes are those described in the previous propositions. Second, we establish a claim which exhibits the necessary and sufficient conditions for the existence of some non-monotonic equilibria. Third, we prove that there are no other non-monotonic equilibria outcomes than those described in the claim. Fourth we show that the condition of the existence of the non-monotonic equilibria are more restrictive that those for the monotonic ones which are not pooling. Fifth, we conclude with the last statement of our proposition.

By definition, a monotonic equilibrium outcome $o$ has to satisfy $o\left(y \mid v_{1}\right) \leq o\left(y \mid v_{2}\right) \leq \ldots \leq o\left(y \mid v_{N}\right)$. So, at a monotonic equilibrium $D M$ 's strategy $\alpha$ has to satisfy $\alpha\left(y \mid r=v_{1}\right) \leq \alpha\left(y \mid r^{\prime}\right)$ for any report $r^{\prime}<q^{E}$. Otherwise, from the optimality of E's strategy we would have $o\left(y \mid v_{1}\right)>o(y \mid v)$ for any value $v \in\left[r^{\prime}, q^{E}\right)$. At any value $v<q^{E}$, E's optimal report $r^{\prime \prime}$ then satisfies $\alpha(y \mid r=$ $\left.v_{1}\right)=\alpha\left(y \mid r^{\prime \prime}\right)$. By Lemma $\left.1 i\right)$ any value $v>\max \left\{q^{E}, q^{D M}\right\}$ induces the action $y$. Consider the case $q^{D M} \leq q^{E}$. Depending on $\alpha\left(y \mid r=v_{1}\right)$ we then find either the equilibrium outcome described in Proposition 1 (if $\alpha\left(y \mid r=v_{1}\right)=1$ ), or those described in Proposition 2 (the first one if $\alpha\left(y \mid r=v_{1}\right)=0$, and the second one (in the footnote) if $\left.\alpha\left(y \mid r=v_{1}\right) \in(0,1)\right)$. In the case $q^{E} \leq q^{D M}$ by Lemma $2 i$ ) there is no optimal DM's strategy which is mixed. So, from the monotonicity of the equilibrium there exists a threshold $q \in\left[v_{1}, v_{N}\right]$ such that any value $v<q$ (if it exists) induces the action $x$ and any value $v>q$ induces the action $y$. If $q=v_{1}$, we find the equilibrium outcome described in Proposition 1. Otherwise, this threshold $q$ must belong to $\left[q^{E}, q^{D M}\right]$ and we find the equilibria outcomes described in Proposition 3. Therefore all other equilibria outcomes than those exhibited in the three first propositions are non-monotonic.

Claim. There exists a continuum of non-monotonic equilibria, parameterized by $q^{\prime} \leq$ $\min \left\{q^{E}, q^{D M}\right\}$ which induce the action $x$ when $v \in\left[q^{\prime}, q^{E}\right], q^{\prime}>v_{1}$, and the action $y$ else iff the DM's preferred action according to $E\left(v \mid v \in\left[q^{\prime}, q^{E}\right]\right)$ is $x$ and his preferred action according to $E\left(v \mid v \notin\left[q^{\prime}, q^{E}\right]\right)$ is $y .^{4}$

Proof of the Claim. Sufficiency. Suppose that the DM's preferred action according to $E\left(v \mid v \in\left[q^{\prime}, q^{E}\right]\right)$ is $x$ and his preferred action according to $E\left(v \mid v \notin\left[q^{\prime}, q^{E}\right]\right)$ is $y$. The following players' strategies support the equilibria described in the claim. An expert of type $v$ reports $r=v_{1}$ if $v \notin\left[q^{\prime}, q^{E}\right]$ and reports $r_{\text {min }}=\min \left\{v_{i} \in\left\{v_{1}, v_{2}, \ldots, v_{N}\right\} \mid v_{i}>q^{\prime}\right\}$ else. $D M$ chooses the action $x$ if $r=r_{\min }$ and the action $y$ else.

Necessity. Suppose there exists an equilibrium, parameterized by $q^{\prime} \leq \min \left\{q^{E}, q^{D M}\right\}, q^{\prime}>v_{1}$, which induces the action $x$ when $v \in\left[q^{\prime}, q^{E}\right]$ and the action $y$ else whereas $D M$ 's preferred action according to $E\left(v \mid v \in\left[q^{\prime}, q^{E}\right]\right)$ is not $x$ and/or $D M$ 's preferred action according to $E(v \mid v \notin$ $\left[q^{\prime}, q^{E}\right]$ ) is not $y$. Under this equilibrium, all reports sent (with positive probabilities) by an expert with value $v \in\left[q^{\prime}, q^{E}\right]$ must be different than those sent by an expert with value $v \notin\left[q^{\prime}, q^{E}\right]$. So, after has received a report, $D M$ learns whether $v \in\left[q^{\prime}, q^{E}\right]$ or not. Now, since either $D M$ 's preferred action according to $E\left(v \mid v \in\left[q^{\prime}, q^{E}\right]\right)$ is not $x$ and/or $D M$ 's preferred action 
according to $E\left(v \mid v \notin\left[q^{\prime}, q^{E}\right]\right)$ is not $y$, the martingale property implies that there must exists a value $v$ under which with positive probability the expert makes a report to which $D M$ 's best responds not $x$ if $v \in\left[q^{\prime}, q^{E}\right]$ and/or not $y$ else, a contradiction.

Now, let us prove that all non-monotonic equilibria outcomes are those described in the claim. By Lemma $1 i$ ) any value $v>\max \left\{q^{E}, q^{D M}\right\}$ induces the action $y$. Consider the case $q^{E} \leq q^{D M}$. By Lemma $2 i$ ) there is no mixed equilibrium. That is, any $v$ induces either the action $x$ or $y$. From the non-monotonicity of the equilibrium, there exist two values $\tilde{v}_{1}$ and $\tilde{v}_{2}$ which respectively induce the action $y$ and $x$ and satisfying $\tilde{v}_{1}<\tilde{v}_{2}$. From the optimality of E's strategy, we then have $\tilde{v}_{2}<q^{E}$ and any $v \in\left[\tilde{v}_{2}, q^{E}\right]$ induces the action $x$. Therefore, as established in the claim, there exists a threshold $q^{\prime}=\min \{v \mid v$ induces the action $x\}$ such that any $v \in\left[q^{\prime}, q^{E}\right]$ induces the action $x$ while any other $v$ induces the action $y$. Now, consider the case $q^{E}>q^{D M}$. From the non-monotonicity of the equilibrium, there exist two values $\tilde{v}_{1}$ and $\tilde{v}_{2}, \tilde{v}_{1}<\tilde{v}_{2}<q^{E}$, which induce the action $y$ with respectively probability $\beta_{1}$ and $\beta_{2}, \beta_{1}>\beta_{2}$. By Lemma 2 ii) if $\beta_{1}<1$ then $\beta_{2}=0$, and if $\beta_{2}>0$ then $\beta_{1}=1$. Consider the case where $\beta_{2}>0$ and $\beta_{1}=1$. Any $v \in\left[\tilde{v}_{2}, q^{E}\right]$ must induces the action $y$ with probability $\beta_{2}$. Otherwise, if there exists a value $v^{\prime} \in\left[\tilde{v}_{2}, q^{E}\right]$ which induces the action $x$ then we either have that any value who induces the action $x$ is higher than $q^{D M}$ or that any value who induces the action $y$ with probability $\beta_{2}$ is lower than $q^{D M}$. But this together with the optimality of DM's strategy leads to a contradiction. Therefore, as established in (the footnote of) the claim, there exists a threshold $q^{\prime}=\min \left\{v \mid v\right.$ induces the action $y$ with probability $\left.\beta_{2}\right\}$ such that any $v \in\left[q^{\prime}, q^{E}\right]$ induces the action $y$ with probability $\beta_{2}$ while any other $v$ induces the action $y$. Considering the case where $\beta_{1}<1$ and $\beta_{2}=0$ leads to a contradiction. Indeed, as previously, we could establish the existence of a threshold $q^{\prime}=\min \{v \mid v$ induces the action $x\}$ such that any $v \in\left[q^{\prime}, q^{E}\right]$ induces the action $x$. Since any $v>q^{E}$ induces the action $y$, the optimality of DM's strategy involves that $\beta_{1}=1$.

Step four, let us show that the conditions exhibited in the claim for the existence of the nonmonotonic equilibria are more restrictive than those exhibited in Proposition 3 and Proposition 2. Assume $q^{D M} \leq q^{E}$. Fix a $q^{\prime} \leq q^{D M}$. It is easily seen that if DM's preferred action according to $E\left(v \mid v \in\left[q^{\prime}, q^{E}\right]\right)$ is $x$ then $x$ is also his preferred action according to $E\left(v \mid v \leq q^{E}\right.$ ) (as in Proposition 2). Now, assume $q^{E} \leq q^{D M}$. Fix a $q^{\prime} \leq q^{E}$. If DM's preferred action according to $E\left(v \mid v \notin\left[q^{\prime}, q^{E}\right]\right)$ is $y$ then $y$ is also his preferred action according to $E\left(v \mid v>q^{E}\right)$. Observe that this latter condition, namely DM's preferred action according to $E\left(v \mid v>q^{E}\right)$ is $y$, is the more restrictive condition on the parameter $q$ exhibited in Proposition 3.

Finally, remark that the footnote of Proposition 2 deals with a mixed equilibrium outcome which generically does not exist since it existence requires that $q^{D M}=E\left(v \mid v<q^{E}\right)$. So by comparing the equilibrium exhibited in Proposition 2 and Proposition 3 with those exhibited in the claim, we trivially have that DM is generically better-off ex-post (resp. ex-ante) in any monotonic equilibrium which is not pooling than in any pure (resp. mixed) non-monotonic one. 


\section{NOTES}

\footnotetext{
${ }^{1}$ In this paper, the terms "verifiable", "certifiable" and "provable" are equivalent.

${ }^{2}$ Although the authors study a situation with several experts, the full revelation of information does not rely on the experts' competition (contrary to Lipman and Seppi (1995)).

${ }^{3}$ The special case where according to $E\left(v \mid v<q^{E}\right)$ DM is indifferent between both actions $x$ and $y$ is a necessary and sufficient condition to the existence of some (mixed) equilibria which induce the action $y$ when $v>q^{E}$ and induce the action $y$ with a fixed probability else.

${ }^{4}$ The special case where the DM's best response to $E\left(v \mid v \in\left[q^{\prime}, q^{E}\right]\right)$ is both actions $x$ and $y$ is a necessary and sufficient condition to the existence of some (mixed) equilibria where when $v \in\left[q^{\prime}, q^{E}\right]$ the action $y$ is induced with a (fixed) probability instead.
}

\section{REFERENCES}

Giovannoni, F. and Seidmann, D.J.: (2006), Secrecy, Two-Sided Bias and the Value of Evidence, Games and Economic Behavior, forthcoming.

Grossman, S.J.: (1981), The informational role of warranties and private disclosure about product quality, Journal of Law and Economics 24(3): 461-483.

Grossman, S.J. and Hart, O.D.: (1980), Disclosure laws and takeover bids, Journal of Finance 35: 323-334.

Koessler, F.: (2003), Persuasion games with higher-order uncertainty, Journal of Economic Theory 110: 393-399.

Lipman, B.L. and Seppi, D.J.: (1995), Robust Inference in Communication Games with Partial Provability Journal of Economic Theory 66: 370-405.

Mathis, J.: (2007), Full Revelation of Information in Sender-Receiver Games of Persuasion, mimeo.

Milgrom, P.: (1981), Good news and bad news: representation theorems and applications, Bell Journal of Economics 12: 380-391.

Milgrom, P. and Roberts, J.: (1986), Relying on the Information of Interested Parties, $\underline{\text { RAND Journal }}$ of Economics 17: 18-32.

Seidmann, D.J. and Winter, E.: (1997), Strategic Information Transmission with Verifiable Messages, Econometrica 65: 163-170.

Shin, H.S.: (1994), The Burden of Proof in a Game of Persuasion, Journal of Economic Theory 64: 253-264.

Shin, H.S.: (1994), News Managements and the Value of Firms, RAND Journal of Economics 25: 58-71.

Wolinsky, A.: (2002), Eliciting information from multiple experts, Games and Economic Behavior 41: 141-160.

Wolinsky, A.: (2003), Information transmission when the sender's preferences are uncertain, Games and Economic Behavior 42: 319-326.

Address for correspondence: T. Lanzi, Lille School of Management, Paris Campus, Tour la Villette, 6, rue Emile Reynaud, 75019 Paris, France. E-mail address: t.lanzi@esc-lille.fr

J. Mathis, Université de Toulouse I, GREMAQ (UMR CNRS 5604), Aile J.J. Laffont, 21 Allée de Brienne, 31000 Toulouse, France. E-mail address: jerome.mathis@univ-tlse1.fr 